\title{
A POSSIBLE CAUSE FOR THE DIELECTRIC FAILURE OF PORCELAINS WHICH ARE APPARENTLY FREE FROM MECHANICAL DEFECTS.
}

\author{
PRELIMINARY DISCUSSION.
}

By Chester Treischel.

From the first uses of porcelain as an insulation in high voltage transmission, difficulties have frequently resulted from the failure of insulators which visually appeared satisfactory, and electrical and ceramic engineers have endeavored to find the cause of these failures and an explanation of porcelain apparently losing its initial dielectric strength without apparent mechanical change.

One of the most widely accepted explanations of this breakdown phenomena is that analogy known as "hammering effect" of the current at high potentials. This analogy, however, becomes somewhat abstract when one asks for a definition of the term "hammering effect." It would seem then that a theory founded on the concrete instead of an analogy founded on the abstract would be a better explanation of electrical failure and would be of greater value to the scientist.

For a number of years it has been known that if crystals of certain substances, such as quartz, are subjected to a pressure on two diametrically opposite faces, these faces being parallel to the major axes, a measurable potential difference is set up, and that this potential difference varies directly as the pressure. This phenomena, known as the piezo-electric effect, was described by Lord Kelvin in two articles appearing in the Philosophical Magazine, 36 ( 1893 ).

The converse of this phenomena has been found to hold true, namely, that if a potential difference is set up on two opposite faces of a crystal, the sides being parallel to the major axis, the crystal is subjected to a squeezing action.

The application of such a phenomena in explaining the electrical failure of porcelain is at once apparent. Porcelain contains large 
quantities of quartz crystals, this fact having been determined microscopically, the crystals being combined together by a glassy magma of fused feldspar. These crystals have no definite position with respect to the direction of their major axes.

Now then, what happens when we subject this porphyritic structure to the influence of alternating high potential? The theory of probabilities will uphold the statement that a number of these quartz crystals are arranged so that their major axes are at right angles to the direction of the flow of the electric current. The current itself is changing its polarity in accordance with the cyclic change, and during each cyclic change there are points of maximum and zero potential difference. If the current is of 60 cycles there will be I 20 maximum potential differences and 120 zero potential differences per second. The effect on each individual crystal, which is arranged with its axis in the right direction, will be a vibratory movement parallel to the direction of the flow of the displacement current. This effect could cause breakdown of the dielectric in three different manners : first, a rupturing of the crystals, second, a rupturing of the glassy magma surrounding the crystals; and third, a leakage of current through the voids between the crystals and the magma when the crystal is at its greatest deformation and the potential difference is at its maximum.

RESEARCH LABORATORY,

General Ei,ectric Company,

SCHENECTADY, NEW YORK.

\section{DISCUSSIONS.}

Albert PECK: While the writer is aware that in many minerals, possessing so-called polar axes, elastic deformation will set up a difference in potential at the opposite ends of the axes, and that conversely a difference in potential along a polar axis will elastically deform the crystal, he does not feel familiar enough with the phenomena to offer other than a few suggestions which might serve as starting points for further investigation.

In the first place, experiments of the type just mentioned are usually performed on perfectly developed crystals or those artificially produced by grinding. Quartz in porcelain is always present in irregular fragments and never in good crystals so that 
if the above principle will not hold for properly oriented irregular fragments, the theory cannot hold. It would seem, however, that the outward form of the quartz is immaterial since we are concerned with directions only. While there undoubtedly would be a few fragments properly oriented with their major axes lying in a plane perpendicular to the direction of the flow of the current, there are also an almost infinite number of possibilities that the greater part are not oriented correctly. It, therefore, becomes a question of whether under the most favorable circumstances these properly oriented crystals would be present in large enough numbers to have sufficient effect to cause failure of the entire body. It is assumed that the author means that the vibratory effect gradually fatigues the fragments until complete rupture of the fragments results. If the fragments do shatter in this way, it seems possible that this could be detected by a microscopic examination of a thin section of porcelain which has failed - as we can similarly observe the shattering of quartz fragments in silica brick.

It is difficult to conceive of a decided change in specific gravity taking place in the quartz without inversion to some other form of silica. While contraction occurs along the axis in the direction of the pressure, there are also two other axes of like character and it seems probable that the contraction in the direction of one axis would be taken up by a corresponding expansion in the direction of the others and equally divided between them so that the volume would not change. Instead of conceiving of a greater density for the fragment, is it not easier to imagine that the fragment may contract in one direction and expand in others against the resistance of the feldspar bond, thus leaving space on one side where the current might leak through?

In passing, attention might be called to the effect of any heating which the porcelain may experience through induction or other electrical effects. Even slight increases in temperature may bring about electrolytic action through the feldspar matrix which rapidly lowers the dielectric strength and electrical resistance of the body. 
E. W. WASHBURN: Since the breakdown of the insulator occurs at comparatively moderate temperatures, it would seem to be improbable that any such explanation as that offered by the author is likely to correspond to the facts-owing to the extremely high viscosity and the corresponding difficulty of moving the crystals in the porcelain body.

If the explanation is correct, a microscopic comparison of the body before and after the breakdown ought to yield confirmatory evidence.

UNIVERSITY OF ILLINOIS,

URBANA, ILLINOIS.

W. R. GILDARD: In the burning of porcelain the flux attacks the quartz, taking a part of it into solution while the balance is more or less corroded, as may be seen by a microscopic examination in thin section. This solution is effected by what we may call "washing," firmly embedding the crystal in a matrix of varying silica content. As the crystal is being dissolved there is not the sharp line of demarcation as would be the case if the substance were insoluble (as an illustration, a piece of iron embedded in ice). It appears to the writer that the formation of a void of even microscopic proportions when the crystal is shortened is questionable, but rather that an additional stress is set up in tension.

The theory advanced appears to be an explanation of the "fatigue" and "hammering effects" of the electrical engineers.

According to the work of the Messrs. Curie and of Lord Kelvin, on this piezo-electrical property of quartz, the potential difference is sufficiently large to permit the truth of this proposed explanation being demonstrated in an experimental way.

RESEARCH LABORATORY,

Generai, Ei,ectric Company,

SCHENECTADY, NEW YORK.

SUBSEQUENT DISCUSSION.

Chester Treischel: The uses of a theory, in the order of their importance, are:

I. It is useful in explaining facts as they are.

2. It is useful in forming a basis for the deduction of further facts. 
3. It is useful in that it gives intellectual satisfaction.

Granting the above, what facts or phenomena will the theory of the vibration of the quartz crystals in porcelain explain? In what way might it form a basis for the deduction of further facts and for the carrying on of further experimental work?

To the writer it seems that this theory explains the following four fundamental facts:

I. That the substitution of certain substances such as talc, pyrophyllite, sillimanite, calcine, etc., for the quartz in an electrical porcelain, increases the dielectric strength of that porcelain.

2. That porcelains high in feldspar and low in quartz content are stronger dielectrically than those of low feldspar and high quartz content, the amount of clay used in either case being the same.

3. 'That porcelains tested with high frequency and with low frequency current at the same high potential, show that the disruptive effect of the high frequency current is much greater than that of the low frequency current.

4. That porcelains tested with direct or alternating currents at the same potentials show that the alternating current has a much greater disruptive effect than the direct current.

The replacement of the quartz in electrical porcelain with various substances has been attempted many times in an experimental way. In every case with which the writer is familiar, such a replacement has resulted in an increased dielectric strength. Even iron oxide in small quantities produced gratifying results. In this case, the amount of quartz present is materially reduced by the substitution of some substance for it. This substance being inert piezo-electrically, there of course is no crystal oscillation during the electrical test and the result is a porcelain of greater dielectric strength.

The same is true of the second case except that we do not have a complete substitution, but rather a partial replacement of quartz by feldspar. The piezo-electric effect, however, applies equally as well since there is a smaller number of quartz crystals present and consequently a smaller number of them properly oriented. 
In explaining the greater disruptive effect of high frequency current over low frequency current we have in the case of the high frequency a greater number of pulsations per second than with the sixty cycle current. This no doubt fatigues the crystals more rapidly, causing a quicker rupture, just as in the case of the rapid and slow bending of a piece of steel we have the rapid bending causing the damage first.

With the use of direct current we have something of the same effect as that noted with the use of low frequency, the frequency here being zero. Of course, there is no vibration of the crystal in this case, merely a squeezing action - consequently a less violent effect upon the porcelain and noticeably greater dielectric strength than with the alternating current.

Now then, in what fields of further research will this theory lead us? It seems to the writer that there are two large and important ones, viz.:

I. The development of a substitute for flint which will not interfere with the proper working and burning of the porcelain batch. In other words, we want a material which has all of the good qualities of flint and enough inherent good qualities of its own to overshadow the bad qualities of flint.

2. The development of a feldspar substitute which will have all of the desirable fluxing and dielectric properties of feldspar but at the same time will be more elastic and less brittle. 